\title{
Dessecação de Plantas Daninhas com Glyphosate em Mistura COM UREIA OU SUlfato DE AMÔNIO ${ }^{1}$
}

\author{
Weed Desiccation With Glyphosate Mixed With Urea or Ammonium Sulfate \\ CARVALHO, S.J.P. ${ }^{2}$, DAMIN, V. ${ }^{3}$, DIAS, A.C.R. ${ }^{2}$, MELO, M.S.C. ${ }^{4}$, NICOLAI, M. ${ }^{2}$ e \\ CHRISTOFFOLETI, P.J. ${ }^{5}$
}

\begin{abstract}
RESUMO - O glyphosate é um herbicida não-seletivo, sistêmico, usado para controle de plantas daninhas anuais e perenes em todo o mundo. A absorção da molécula do glyphosate ocorre pelos tecidos fotossinteticamente ativos das plantas, porém alguns fatores podem reduzir sua eficácia, como a morfologia e diversidade de espécies, chuva após aplicação, qualidade da água e misturas em tanque com outros defensivos, entre outros. Objetivouse com este trabalho avaliar a influência da adição de sulfato de amônio ou ureia em calda na eficácia do herbicida glyphosate para dessecação de plantas daninhas. Dois experimentos foram desenvolvidos em Piracicaba - SP, com aplicações de glyphosate (720 e $\left.1.440 \mathrm{~g} \mathrm{ha}^{-1}\right)$ isolado ou combinado com duas doses de sulfato de amônio $\left(7,5\right.$ e $\left.15,0 \mathrm{~g} \mathrm{~L}^{-1}\right)$ ou ureia $(2,5$ e 5,0 $\mathrm{g} \mathrm{L}^{-1}$ ) sobre as plantas daninhas: apaga-fogo (Alternanthera tenella) e capim-massambará (Sorghum halepense). Para a espécie menos suscetivel ao herbicida (capim-massambará), a adição de fontes nitrogenadas à menor dose de glyphosate acelerou a morte das plantas, elevando os niveis de controle em até $7,3 \%$ na avaliação de 21 dias após aplicação (DAA) dos tratamentos. Contudo, os efeitos não foram observados nas avaliações de controle, massa fresca e seca, conduzidas aos 28 DAA. A dose recomendada de glyphosate para cada espécie proporcionou controle satisfatório, sem a necessidade de adição de sulfato de amônio ou ureia.
\end{abstract}

Palavras-chave: Sorghum halepense, Alternanthera tenella, eficácia, manejo.

\begin{abstract}
Glyphosate is a non-selective systemic herbicide used to control annual and perennial weeds worldwide. Molecule absorption occurs through the plant's photosynthetically-active tissues; however, some factors might reduce its efficacy, such as morphology and specific diversity, rain after application, water quality and tank mixtures with other chemicals. Thus, this work aimed to evaluate the influence of ammonium sulfate or urea addiction to spray tank on glyphosate efficacy for weed desiccation. Two trials were carried out in Piracicaba-SP, with applications of glyphosate (720 and $1440 \mathrm{~g} \mathrm{ha}^{-1}$ ) alone or combined with two rates of ammonium sulfate (7.5 and $\left.15.0 \mathrm{~g} \mathrm{~L}^{-1}\right)$ or urea (2.5 and $\left.5.0 \mathrm{~g} \mathrm{~L}^{-1}\right)$, over the weeds Alternanthera tenella and Sorghum halepense. For the least susceptible species (S. halepense), the addition of nitrogen sources to the lower rate of glyphosate accelerated plant death, increasing the control levels up to $7.3 \%$, at 21 days after application (DAA). However, the effects were not observed when control, fresh and dry mass were evaluated at $28 \mathrm{DAA}$. Glyphosate recommended rate for each species promoted appropriate control, without the need to add ammonium sulfate or urea.
\end{abstract}

Keywords: Sorgum halepense, Alternanthera tenella, efficacy, management.

1 Recebido para publicação em 21.7.2008 e na forma revisada em 5.6.2009.

Parte da tese do primeiro autor a ser apresentada à ESALQ para obtenção do título de Doutor em Agronomia.

2 Pós-Graduandos em Fitotecnia-ESALQ/USP, <sjpcarvalho@yahoo.com.br>, <acrdias@esalq.usp.br>, <marcelon@esalq.usp.br>; ${ }^{3}$ Doutoranda em Solos e Nutrição de Plantas - ESALQ/USP, <virginia@esalq.usp.br>; ${ }^{4}$ Graduando em Engenharia Agronômica ESALQ/USP, ${ }^{5}$ Professor Associado do Dep. de Produção Vegetal - ESALQ/USP, <pjchrist@esalq.usp.br>, Caixa Postal 09, 13419-900 Piracicaba-SP.

Planta Daninha, Viçosa-MG, v. 27, n. 2, p. 353-361, 2009 


\section{INTRODUÇÃO}

Com o advento das culturas geneticamente modificadas para resistência a herbicidas, a adoção do glyphosate cresceu significativamente nos últimos anos (Young et al., 2003). A adoção de sistemas conservacionistas de manejo de solo (plantio direto, cultivo mínimo, etc.), frequentemente baseados em dessecação pré-semeadura, também contribuiu para a ampla utilização dessa molécula (Christoffoleti et al., 2008), que atualmente é considerada o herbicida de maior importância mundial (Moreira \& Christoffoleti, 2008).

O glyphosate é um herbicida não-seletivo, de ação sistêmica, usado no controle de plantas daninhas anuais e perenes, cuja absorção se dá pelas estruturas fotossinteticamente ativas das plantas. Atua inibindo a enzima enol piruvil shiquimato fosfato sintase (EPSPs), que participa da rota de síntese dos aminoácidos aromáticos fenilalanina, tirosina e triptofano (Rodrigues \& Almeida, 2005). A absorção via cutícula é lenta, sendo necessário período de quatro a seis horas sem chuvas após a aplicação para ocorrer o controle satisfatório de plantas suscetiveis (Jakelaitis et al., 2001; Pedrinho Júnior et al., 2002).

Além da ocorrência de chuvas após aplicação, outros fatores podem resultar na menor eficácia do produto, entre eles: mistura em tanque com outros herbicidas (Costa \& Appleby, 1986); morfologia foliar, absorção, translocação e metabolismo nas plantas-alvo (Norsworthy et al., 2001; Monquero et al., 2004;); e presença de sais na água (Nalewaja \& Matysiak, 1991; Gauvrit, 2003), que potencialmente podem ser minimizados com a adição de fontes nitrogenadas à calda de pulverização. Segundo Nalewaja \& Matysiak (1991), o antagonismo entre glyphosate e cátions ocorre na seguinte ordem: ferro $>$ zinco $>$ cálcio $\geq$ magnésio $>$ sódio $>$ potássio. Por outro lado, Wills \& McWhorter (1985) observaram que sais de cátions monovalentes $\left(\mathrm{NH}_{4}^{+}, \mathrm{K}^{+}\right.$e Na$)$, geralmente, resultaram em maior toxicidade do glyphosate, enquanto sais de cátions bi ou trivalentes $\left(\mathrm{Zn}^{++}\right.$ e $\mathrm{Fe}^{+++}$) proporcionaram menor atividade herbicídica.

Trabalhos têm sido desenvolvidos visando quantificar a influência de concentrações de sais na água de pulverização sobre a eficácia do herbicida glyphosate. Mueller et al. (2006) observaram que a eficácia do herbicida glyphosate (420 e $840 \mathrm{~g} \mathrm{ha}^{-1}$ ) foi reduzida quando a concentração de cátions (cálcio ou magnésio) na água foi superior a 250 ppm, porém esse antagonismo foi minimizado com a adição de sulfato de amônio a $2 \%$. Shea \& Tupy (1984), por sua vez, observaram menor controle de trigo (Triticum aestivum) com glyphosate a $100 \mathrm{~g} \mathrm{ha}^{-1}$ quando da presença de 50 ppm de cálcio na solução.

Com a redução do antagonismo proporcionado pela presença de sais na calda de pulverização, há também possibilidade de diminuir a quantidade de glyphosate necessária para controle eficaz das plantas daninhas, além de aumentar a consistência dos resultados (Nalewaja \& Matysiak, 1993). A adição de sulfato de amônio às caldas de aplicação de glyphosate utilizando água livre de agentes contaminantes também pode promover maior eficácia do herbicida, em razão da absorção facilitada nas folhas e membranas; contudo, essa resposta pode ser diferencial entre as espécies (Young et al., 2003).

A temática da adição de sulfato de amônio à calda do herbicida glyphosate tem sido estudada intensamente (Salisbury et al., 1991; Thelen et al., 1995; Ramsdale et al., 2003), no entanto somente alguns trabalhos contemplaram espécies com ocorrência no Brasil. Por outro lado, poucos foram os estudos que avaliaram os efeitos da adição de ureia à calda - prática esta comumente utilizada por produtores brasileiros. Nesse sentido, Durigan (1992) estudou o efeito da adição de adjuvantes na calda de aplicação de glyphosate para controle de capim-colonião (Panicum maximum) e constatou que a adição de $0,2 \%$ de ureia, em volume de calda de $500 \mathrm{~L} \mathrm{ha}^{-1}$, proporcionou redução da dose de glyphosate em $360 \mathrm{~g} \mathrm{ha}^{-1}$.

Este trabalho foi conduzido com o objetivo de caracterizar a influência da adição de sulfato de amônio ou ureia na eficácia do herbicida glyphosate para dessecação de plantas daninhas.

\section{MATERIAL E MÉTODOS}

Dois experimentos foram desenvolvidos em áreas adjacentes do Departamento de Produção Vegetal da Escola Superior de 
Agricultura "Luiz de Queiroz" - ESALQ/USP, em Piracicaba - SP - Brasil (22 42' 30' latitude sul, $47^{\circ} 38^{\prime}$ 00' longitude oeste e $546 \mathrm{~m}$ de altitude), no período entre abril e maio de 2008. O solo de ambas as áreas é classificado como Nitossolo Vermelho eutrófico de textura argilosa, $\mathrm{pH} 5,4$, com $2,5 \%$ de matéria orgânica e cerca de $68 \%$ de saturação por bases. No primeiro experimento, havia na área a predominância de apaga-fogo (Alternanthera tenella) com 30 plantas $\mathrm{m}^{-2}$; já no segundo a espécie predominante foi o capim-massambará (Sorghum halepense), com 20 plantas $\mathrm{m}^{-2}$.

Em ambos os experimentos, o delineamento experimental adotado foi o de blocos ao acaso, com doze tratamentos e três repetições, totalizando 36 parcelas. Cada parcela foi composta por áreas total e útil de 10 e $8 \mathrm{~m}^{2}$, respectivamente. Os tratamentos resultaram da combinação entre doses do herbicida glyphosate (sal de isopropilamina - Roundup Original $^{\circledR}-720$ e $1.440 \mathrm{~g} \mathrm{ha}^{-1}$ ) e das fontes nitrogenadas: ureia $\left(2,5\right.$ e $\left.5,0 \mathrm{~g} \mathrm{~L}^{-1}\right)$ e sulfato de amônio (7,5 e 15,0 $\left.\mathrm{g} \mathrm{L}^{-1}\right)$; acrescidos de um tratamento com herbicida de outro mecanismo de ação (glufosinato de amônio - $400 \mathrm{~g} \mathrm{ha}^{-1}$ ); e testemunha sem aplicação. As doses de glyphosate foram estimadas nas recomendações de registro para as duas espécies (Rodrigues \& Almeida, 2005), enquanto as doses das fontes nitrogenadas foram escolhidas visando comprovação da prática de campo.
Todos os tratamentos estão detalhadamente descritos na Tabela 1 .

As aplicações de herbicidas foram realizadas no dia 4 de abril de 2008, das 14 h às 15 h, em plantas de apaga-fogo com estádio fenológico de pleno florescimento e capim-massambará em pré-florescimento. No momento das aplicações, as médias das condições meteorológicas foram: URA, 81,2\%; T, $25,2{ }^{\circ} \mathrm{C}$; Céu, $50 \%$ encoberto; e ventos de $3,7 \mathrm{~km} \mathrm{~h}^{-1}$. A primeira chuva foi registrada 48 horas após a aplicação. As precipitações e a temperatura média diária do período em que o experimento foi desenvolvido estão apresentadas na Figura 1.

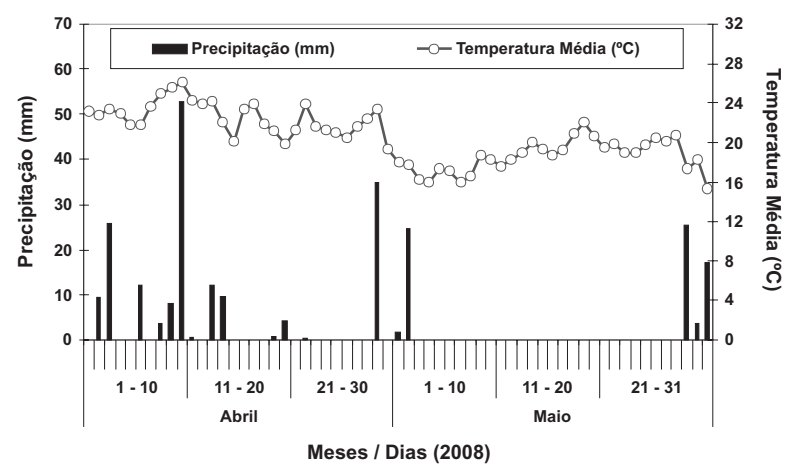

Figura 1 - Temperatura média $\left({ }^{\circ} \mathrm{C}\right)$ e precipitações $(\mathrm{mm})$ diárias observadas durante o período de condução dos experimentos. Piracicaba, 2008.

Tabela 1 - Tratamentos herbicidas aplicados nos dois experimentos. Piracicaba, 2008

\begin{tabular}{|c|c|c|c|c|c|}
\hline \multirow{3}{*}{$\mathrm{N}^{\mathrm{o}}$} & \multicolumn{2}{|c|}{ Herbicida } & \multicolumn{3}{|c|}{ Fertilizante nitrogenado } \\
\hline & \multirow{2}{*}{ Condição } & \multirow{2}{*}{ Dose $\left(\mathrm{g} \mathrm{ha}^{-1}\right)$} & \multirow{2}{*}{ Tipo } & \multicolumn{2}{|c|}{ Dose } \\
\hline & & & & $\left(\mathrm{g} \mathrm{L}^{-1}\right)$ & $\left(\mathrm{kg} \mathrm{ha}^{-1}\right)$ \\
\hline 1 & Ausente & 0 & Ausente & 0,0 & 0,0 \\
\hline 2 & Glyphosate $^{1 /}$ & 720 & Ausente & 0,0 & 0,0 \\
\hline 3 & Glyphosate & 720 & Sulfato de Amônio & 7,5 & 1,5 \\
\hline 4 & Glyphosate & 720 & Sulfato de Amônio & 15,0 & 3,0 \\
\hline 5 & Glyphosate & 720 & Ureia & 2,5 & 0,5 \\
\hline 6 & Glyphosate & 720 & Ureia & 5,0 & 1,0 \\
\hline 7 & Glyphosate & 1.440 & Ausente & 0,0 & 0,0 \\
\hline 8 & Glyphosate & 1.440 & Sulfato de Amônio & 7,5 & 1,5 \\
\hline 9 & Glyphosate & 1.440 & Sulfato de Amônio & 15,0 & 3,0 \\
\hline 10 & Glyphosate & 1.440 & Ureia & 2,5 & 0,5 \\
\hline 11 & Glyphosate & 1.440 & Ureia & 5,0 & 1,0 \\
\hline 12 & \multicolumn{2}{|c|}{ Glufosinato de Amônio ${ }^{2 /}$} & \multicolumn{3}{|c|}{ Ausente } \\
\hline
\end{tabular}

${ }^{1 /}$ Roundup Original ${ }^{\circledR}, 360$ g e.a. L-1; ${ }^{2 /}$ Finale $^{\circledR}, 200$ g L$^{-1}$. 
Para as aplicações, utilizou-se pulverizador costal de precisão pressurizado por $\mathrm{CO}_{2}$, acoplado a uma barra de pulverização com largura útil de $2 \mathrm{~m}$, com quatro pontas de aplicação do tipo jato plano, 110.02, espaçadas de $0,50 \mathrm{~m}$, o que proporcionou a aplicação de $200 \mathrm{~L} \mathrm{ha}^{-1}$ de calda herbicida. A água utilizada nas aplicações procede de tratamento comum para distribuição pública, realizado na própria ESALQ/USP, cuja análise encontra-se na Tabela 2.

As variáveis avaliadas foram: controle percentual aos 7, 14, 21 e 28 dias após a aplicação (DAA) dos tratamentos e massas fresca e seca aos 28 DAA. Nas avaliações de controle utilizou-se escala visual, atribuindo-se notas de zero a $100 \%$, em que zero representou a ausência de sintomas e 100 a morte de todas as plantas. Quanto à coleta das massas fresca e seca, utilizou-se um quadro de madeira de $0,25 \mathrm{~m}^{2}(0,5 \times 0,5 \mathrm{~m})$, o qual foi lançado aleatoriamente em área representativa da parcela em que era realizada a avaliação. Após a coleta da parte aérea do material vegetal, este foi seco em estufa $\left(70^{\circ} \mathrm{C}\right)$ até massa constante, quando se determinaram as massas fresca e seca. Para permitir comparação dos experimentos, a massa seca foi ajustada a termos percentuais, considerando-se $100 \%$ a parcela testemunha de cada bloco.

Procedeu-se à análise por grupo de experimentos, com aplicação do teste $\mathrm{F}$ na análise da variância, seguido da aplicação do teste ' $t$ ' para comparação das espécies (experimentos) e teste de Scott-Knott (Scott \& Knott, 1974) para agrupamento das médias dos tratamentos herbicidas, ambos com 5\% de significância.

\section{RESULTADOS E DISCUSSÃO}

Foi identificada interação dos tratamentos herbicidas com os diferentes experimentos (espécies) para todas as avaliações de controle, porém não houve interação para massas fresca e seca. Aos 7 DAA, constatou-se efeito da dose de glyphosate no controle de apaga-fogo e capim-massambará, em que a aplicação de $1.440 \mathrm{~g} \mathrm{ha}^{-1}$ apresentou maior índice de controle, com diferença média de 10 e $20 \%$ em relação a $720 \mathrm{~g}^{\text {ha }}{ }^{-1}$, para apaga-fogo e capimmassambará, respectivamente. Entre as espécies, o capim-massambará foi identificado como a menos sensivel ao glyphosate, uma vez que o controle obtido com a dose de $720 \mathrm{~g} \mathrm{ha}^{-1}$ foi menor que o obtido para apaga-fogo quando se aplicou o herbicida puro ou combinado com sulfato de amônio (Tabela 3).

A adição de ureia a $2,5 \mathrm{~g} \mathrm{~L}^{-1}$ na calda de glyphosate $\left(720 \mathrm{~g} \mathrm{ha}^{-1}\right)$ resultou em incremento de controle de capim-massambará aos 7 DAA, pois, além de proporcionar igualdade entre as espécies, diferenciou-se do tratamento com glyphosate puro na mesma dose. Por outro lado, para a maior dose de glyphosate, a adição de

Tabela 2 - Análise da água utilizada nas aplicações, submetida a tratamento usual para distribuição pública, realizado na ESALQ/USP. Piracicaba, 2008

\begin{tabular}{|c|c|c|c|c|c|}
\hline Parâmetro & Unidade & Resultado & Parâmetro & Unidade & Resultado \\
\hline Cloreto $\left(\mathrm{Cl}^{-}\right)$ & $\mathrm{mg} \mathrm{L}^{-1}$ & 26,0 & Zinco $(\mathrm{Zn})$ & $\mathrm{mg} \mathrm{L}^{-1}$ & 0,09 \\
\hline Nitrato $\left(\mathrm{N}-\mathrm{NO}_{3}\right)$ & $\mathrm{mg} \mathrm{L}^{-1}$ & 11,5 & Alcalinidade $\left(2 \mathrm{CO}_{3}{ }^{2-}+\mathrm{HCO}_{3}{ }^{-}\right)$ & $\mathrm{mg} \mathrm{L}^{-1}$ & 40,2 \\
\hline Sulfato $\left(\mathrm{SO}_{4}{ }^{2-}\right)$ & $\mathrm{mg} \mathrm{L}^{-1}$ & 68,3 & Nitrogênio amoniacal $\left(\mathrm{N}-\mathrm{NH}_{3}\right)$ & $\mathrm{mg} \mathrm{L}^{-1}$ & 0,2 \\
\hline Fósforo $(\mathrm{P})$ & $\mathrm{mg} \mathrm{L}^{-1}$ & 0,01 & Cor aparente & $\mathrm{PtCo}$ & 0 \\
\hline Sódio $\left(\mathrm{Na}^{+}\right)$ & $\mathrm{mg} \mathrm{L}^{-1}$ & 36,5 & Turbidez & FTU & 0 \\
\hline Potássio $\left(\mathrm{K}^{+}\right)$ & $\mathrm{mg} \mathrm{L}^{-1}$ & 4,8 & Sedimentos em suspensão & $\mathrm{mg} \mathrm{L}^{-1}$ & 1,0 \\
\hline Cálcio $\left(\mathrm{Ca}^{2+}\right)$ & $\mathrm{mg} \mathrm{L}^{-1}$ & 38,8 & Condutividade elétrica (CE) & $\mathrm{mS} \mathrm{cm}^{-1}$ & 0,26 \\
\hline Magnésio $\left(\mathrm{Mg}^{2+}\right)$ & $\mathrm{mg} \mathrm{L}^{-1}$ & 2,7 & $\mathrm{pH}$ & -- & 8,2 \\
\hline Ferro $(\mathrm{Fe})$ & $\mathrm{mg} \mathrm{L}^{-1}$ & 0,02 & Gás carbônico $\left(\mathrm{CO}_{2}\right)$ & $\mathrm{mg} \mathrm{L}^{-1}$ & 0,4 \\
\hline Cobre $(\mathrm{Cu})$ & $\mathrm{mg} \mathrm{L}^{-1}$ & 0 & Acidez $\left(\mathrm{CaCO}_{3}\right)$ & $\mathrm{mg} \mathrm{L}^{-1}$ & 1,0 \\
\hline Manganês (Mn) & $\mathrm{mg} \mathrm{L}^{-1}$ & 0,02 & Dureza total* $\left(\mathrm{CaCO}_{3}\right)$ & $\mathrm{mg} \mathrm{L}^{-1}$ & 107,8 \\
\hline
\end{tabular}

Análises realizadas pelo Laboratório de Ecologia Aplicada, Departamento de Ciências Florestais, ESALQ/USP. * Dureza Total calculada com base no equivalente de Carbonato de Cálcio $\left(\mathrm{CaCO}_{3}\right)$, segundo Franson (1995): 2,497 [Ca, mg L-1] + 4,118 [Mg, $\left.\mathrm{mg} \mathrm{L}^{-1}\right]$. 
Tabela 3 - Controle percentual de apaga-fogo (ALRTE) e capim-massambará (SORHA), avaliado aos 7 e 14 dias após aplicação (DAA) dos tratamentos. Piracicaba, 2008

\begin{tabular}{|c|c|c|c|c|c|c|}
\hline \multirow{3}{*}{ Tratamento } & \multicolumn{2}{|c|}{ Dose } & \multicolumn{4}{|c|}{ Controle percentual* } \\
\hline & Herbicida & FN & \multicolumn{2}{|c|}{$7 \mathrm{DAA}$} & \multicolumn{2}{|c|}{$14 \mathrm{DAA}$} \\
\hline & $\left(\mathrm{g} \mathrm{ha}^{-1}\right)$ & $\left(\mathrm{g} \mathrm{L}^{-1}\right)$ & ALRTE & SORHA & ALRTE & SORHA \\
\hline Testemunha sem aplicação & -- & -- & $0,0 \mathrm{D}$ a & $0,0 \mathrm{D} \mathrm{a}$ & $0,0 \mathrm{C} \mathrm{a}$ & $0,0 \mathrm{C} \mathrm{a}$ \\
\hline Glyphosate $^{1 / 2}$ & 720 & -- & $68,9 \mathrm{~B} \mathrm{a}$ & $53,6 \mathrm{C} \mathrm{b}$ & $92,7 \mathrm{~A} \mathrm{a}$ & $86,0 \mathrm{~B} \mathrm{~b}$ \\
\hline Glyphosate $+\mathrm{SA}^{2 / 1}$ & 720 & 7,5 & $66,1 \mathrm{~B} \mathrm{a}$ & $54,6 \mathrm{C} \mathrm{b}$ & $92,7 \mathrm{~A} \mathrm{a}$ & $87,7 \mathrm{~B} \mathrm{a}$ \\
\hline Glyphosate + SA & 720 & 15,0 & $66,3 \mathrm{~B} \mathrm{a}$ & $53,3 \mathrm{C} \mathrm{b}$ & $91,7 \mathrm{~A} \mathrm{a}$ & $86,7 \mathrm{~B} \mathrm{a}$ \\
\hline Glyphosate + Ureia & 720 & 2,5 & $63,3 \mathrm{~B} \mathrm{a}$ & $66,7 \mathrm{~B} \mathrm{a}$ & $94,3 \mathrm{~A} \mathrm{a}$ & $91,7 \mathrm{~A} \mathrm{a}$ \\
\hline Glyphosate + Ureia & 720 & 5,0 & $61,1 \mathrm{~B} \mathrm{a}$ & $54,5 \mathrm{C} \mathrm{a}$ & $92,7 \mathrm{~A} \mathrm{a}$ & $89,3 \mathrm{~B} \mathrm{a}$ \\
\hline Glyphosate & 1.440 & -- & $75,0 \mathrm{~A} \mathrm{a}$ & $83,8 \mathrm{~A} \mathrm{a}$ & $98,3 \mathrm{~A} \mathrm{a}$ & $97,7 \mathrm{~A} \mathrm{a}$ \\
\hline Glyphosate + AS & 1.440 & 7,5 & $81,2 \mathrm{~A} \mathrm{a}$ & $78,1 \mathrm{~A} \mathrm{a}$ & $95,7 \mathrm{~A} \mathrm{a}$ & $93,0 \mathrm{~A} \mathrm{a}$ \\
\hline Glyphosate + AS & 1.440 & 15,0 & $78,3 \mathrm{~A} \mathrm{a}$ & $75,0 \mathrm{~A} \mathrm{a}$ & $96,7 \mathrm{~A} \mathrm{a}$ & $94,0 \mathrm{~A} \mathrm{a}$ \\
\hline Glyphosate + Ureia & 1.440 & 2,5 & $71,1 \mathrm{~A} \mathrm{a}$ & $76,3 \mathrm{~A} \mathrm{a}$ & $96,7 \mathrm{~A} \mathrm{a}$ & $95,3 \mathrm{~A} \mathrm{a}$ \\
\hline Glyphosate + Ureia & 1.440 & 5,0 & $78,3 \mathrm{~A} \mathrm{a}$ & $72,2 \mathrm{~B} \mathrm{a}$ & $96,0 \mathrm{~A} \mathrm{a}$ & $94,3 \mathrm{~A} \mathrm{a}$ \\
\hline Glufosinato de Amônio & 400 & -- & $51,1 \mathrm{C} \mathrm{b}$ & $66,1 \mathrm{~B} \mathrm{a}$ & $41,6 \mathrm{~B} \mathrm{~b}$ & $85,0 \mathrm{~B} \mathrm{a}$ \\
\hline \multicolumn{3}{|c|}{$\mathrm{F}_{(\text {Trat x Exp) }}$} & \multicolumn{2}{|c|}{$2,99 * *$} & \multicolumn{2}{|c|}{$19,62 * *$} \\
\hline \multicolumn{3}{|c|}{$\mathrm{DMS}_{\mathrm{Esp}}$} & \multicolumn{2}{|c|}{10,68} & \multicolumn{2}{|c|}{6,14} \\
\hline \multicolumn{3}{|c|}{$\mathrm{CV}(\%)$} & \multicolumn{2}{|c|}{10,41} & \multicolumn{2}{|c|}{4,49} \\
\hline
\end{tabular}

${ }^{1 /}$ Dose de glyphosate, em g ha ${ }^{-1} ;{ }^{2} / \mathrm{SA}$ - sulfato de amônio; * Médias seguidas por letras iguais, maiúsculas nas colunas e minúsculas nas linhas, não diferem entre si pelo teste de agrupamento de médias de Scott-Knott (coluna) ou teste ' $t$ ' (linha), a 5\% de significância; ** Teste $F$ significativo a $1 \%$.

ureia a $5 \mathrm{~g} \mathrm{~L}^{-1}$ resultou em menor controle, indicando possivel antagonismo quando do uso de doses mais concentradas do produto (Tabela 3).

Resultados semelhantes foram observados aos 14 DAA (Tabela 3). Nessa avaliação, para o apaga-fogo, novamente não foram observados efeitos da adição de fontes nitrogenadas à calda do herbicida, o que pode estar relacionado com a aplicação de doses iguais ou superiores à recomendada para a espécie (Rodrigues \& Almeida, 2005). Quanto ao capim-massambará, constatou-se incremento de controle com a aplicação de glyphosate (720 $\mathrm{g}_{\text {ha }}{ }^{1}$ ) acrescido de $2,5 \mathrm{~g} \mathrm{~L}^{-1}$ de ureia. Houve diferenciação das espécies apenas quando se aplicou glyphosate a $720 \mathrm{~g} \mathrm{ha}^{-1}$. A adição de quaisquer doses de sulfato de amônio ou ureia permitiu a igualdade dos niveis de controle das espécies, o que sugere efeito das fontes nitrogenadas (Tabela 3).

Com relação à ureia, Durigan (1992) relata tratar-se de uma substância que penetra com relativa facilidade na cutícula das folhas. Mesmo sem receber energia metabólica, passa do apoplasto para o simplasto foliar por difusão facilitada, resultante do acúmulo de energia cinética pelas moléculas. Segundo esse autor, a ureia também pode romper algumas ligações éster, éter e diéter da cutina, o que contribui para maior absorção de outros elementos presentes na calda, em consequência do aumento dos espaços de entrada. Suwunnamek \& Parker (1975) observaram que a adição de ureia, fosfato-butil-amônio e fosfato de amônio também elevou o controle de tiririca (Cyperus rotundus) pelo glyphosate, porém não apresentou vantagens em relação ao sulfato de amônio.

Salisbury et al. (1991) também estudaram a adição de sulfato de amônio à calda de pulverização para incremento de controle de capimmassambará com o herbicida glyphosate. Esses autores constataram que a adição de sulfato de amônio $\left(3,33 \mathrm{~kg} \mathrm{ha}^{-1}\right)$ à calda de $420 \mathrm{~g} \mathrm{ha}^{-1}$ de glyphosate proporcionou controle comparável ao da dose de $840 \mathrm{~g}$ ha ${ }^{1}$ e que efeitos antagônicos só foram observados com doses de sulfato de amônio superiores a $9,7 \mathrm{~kg} \mathrm{ha}^{-1}$. Hall et al. (2000), por sua vez, não observaram incremento significativo no controle de capimmassambará em decorrência da adição de sulfato de amônio à calda de pulverização. Para caldas oriundas da adoção de água dura (altos níveis de cálcio e magnésio), a maior eficácia do glyphosate em presença do sulfato de 
amônio tem sido explicada, entre outras teorias, pela ligação do $\mathrm{Ca}^{++}$com o ânion sulfato oriundo do fertilizante nitrogenado, resultando em precipitados de $\mathrm{CaSO}_{4}$. Com a remoção do cálcio, o ion $\mathrm{NH}_{4}^{+}$pode se ligar ao glyphosate, gerando um sal que atravessa mais facilmente a cutícula (Thelen et al., 1995).

Os resultados positivos da adição de fontes nitrogenadas à calda do herbicida glyphosate foram evidentes na avaliação de controle conduzida aos 21 DAA (Tabela 4). Entre os tratamentos herbicidas, observou-se baixo controle do capim-massambará para aplicação da menor dose de glyphosate. Para essa espécie, a adição de quaisquer doses de ureia e sulfato de amônio à menor dose de glyphosate proporcionou igualdade desta à maior dose e, também, igualdade de controle entre as espécies, com incrementos de até $7,3 \%$. Também na avaliação realizada aos 21 DAA, o capim-massambará manteve-se como espécie menos suscetível ao glyphosate, o que está de acordo com as doses recomendadas por Rodrigues \& Almeida (2005).

Vale destacar que no momento da aplicação dos herbicidas não havia sedimentos ou excessiva quantidade de poeira sobre as folhas, devido à ocorrência de chuva na véspera. Ainda, considerando-se que não ocorreu chuva logo após a aplicação, que as condições meteorológicas foram adequadas à eficácia dos herbicidas (Figura 1) e que a água utilizada nas aplicações não continha níveis elevados de cálcio, magnésio, ferro ou dureza total (Tabela 2), os incrementos provenientes da adição de fontes nitrogenadas à calda devem estar relacionados com outras vias de resposta que não a redução da atividade de cátions antagônicos.

Nurse et al. (2008) observaram que a adição de sulfato de amônio $(2 \%)$ à calda com água livre de impurezas proporcionou maior eficácia do glyphosate sobre algumas espécies de plantas daninhas, porém somente para doses inferiores às recomendadas, o que está em concordância com os dados avaliados neste traba1ho. Uma das teorias para explicar os incrementos de controle sugere que a adição de sulfato de amônio à calda de glyphosate altera a morfologia das gotas, atrasando ou prevenindo a cristalização do glyphosate na superficie foliar. Dessa forma, há maior tempo para que a molécula atravesse a cutícula (MacIsaac et al., 1991).

Outros estudos demonstraram que o aumento da eficácia do glyphosate proporcionada

Tabela 4 - Controle percentual de apaga-fogo (ALRTE) e capim-massambará (SORHA), avaliado aos 21 e 28 dias após aplicação (DAA) dos tratamentos. Piracicaba, 2008

\begin{tabular}{|c|c|c|c|c|c|c|}
\hline \multirow{3}{*}{ Tratamento } & \multicolumn{2}{|c|}{ Dose } & \multicolumn{4}{|c|}{ Controle percentual* } \\
\hline & Herbicida & $\mathrm{FN}$ & \multicolumn{2}{|c|}{$21 \mathrm{DAA}$} & \multicolumn{2}{|c|}{$28 \mathrm{DAA}$} \\
\hline & $\left(\mathrm{g} \mathrm{ha}^{-1}\right)$ & $\left(\mathrm{g} \mathrm{L}^{-1}\right)$ & ALRTE & SORHA & ALRTE & SORHA \\
\hline Testemunha sem aplicação & -- & -- & $0,0 \mathrm{C} \mathrm{a}$ & $0,0 \mathrm{D} \mathrm{a}$ & $0,0 \mathrm{C} \mathrm{a}$ & $0,0 \mathrm{C} \mathrm{a}$ \\
\hline Glyphosate ${ }^{1 /}$ & 720 & -- & $97,3 \mathrm{~A} \mathrm{a}$ & $90,7 \mathrm{~B} \mathrm{~b}$ & $97,3 \mathrm{~A} \mathrm{a}$ & $95,7 \mathrm{~A} \mathrm{a}$ \\
\hline Glyphosate $+\mathrm{SA}^{2} \stackrel{2}{\prime}$ & 720 & 7,5 & $96,7 \mathrm{~A} \mathrm{a}$ & $95,7 \mathrm{~A} \mathrm{a}$ & $96,3 \mathrm{~A} \mathrm{a}$ & $98,0 \mathrm{~A} \mathrm{a}$ \\
\hline Glyphosate + SA & 720 & 15,0 & $96,7 \mathrm{~A} \mathrm{a}$ & $93,3 \mathrm{~A} \mathrm{a}$ & $97,0 \mathrm{~A} \mathrm{a}$ & $96,7 \mathrm{~A} \mathrm{a}$ \\
\hline Glyphosate + Ureia & 720 & 2,5 & $97,0 \mathrm{~A} \mathrm{a}$ & $98,0 \mathrm{~A} \mathrm{a}$ & $97,0 \mathrm{~A} \mathrm{a}$ & $98,0 \mathrm{~A} \mathrm{a}$ \\
\hline Glyphosate + Ureia & 720 & 5,0 & $97,3 \mathrm{~A} \mathrm{a}$ & $94,3 \mathrm{~A} \mathrm{a}$ & $96,7 \mathrm{~A} \mathrm{a}$ & $97,7 \mathrm{~A} \mathrm{a}$ \\
\hline Glyphosate & 1.440 & -- & $98,7 \mathrm{~A} \mathrm{a}$ & $97,0 \mathrm{~A} \mathrm{a}$ & $98,7 \mathrm{~A} \mathrm{a}$ & $98,0 \mathrm{~A} \mathrm{a}$ \\
\hline Glyphosate + AS & 1.440 & 7,5 & $98,3 \mathrm{~A} \mathrm{a}$ & $97,3 \mathrm{~A} \mathrm{a}$ & $97,3 \mathrm{~A} \mathrm{a}$ & $98,7 \mathrm{~A} \mathrm{a}$ \\
\hline Glyphosate + AS & 1.440 & 15,0 & $98,0 \mathrm{~A} \mathrm{a}$ & $96,7 \mathrm{~A} \mathrm{a}$ & $97,0 \mathrm{~A} \mathrm{a}$ & $98,3 \mathrm{~A} \mathrm{a}$ \\
\hline Glyphosate + Ureia & 1.440 & 2,5 & $97,7 \mathrm{~A} \mathrm{a}$ & $96,7 \mathrm{~A} \mathrm{a}$ & $98,3 \mathrm{~A} \mathrm{a}$ & $97,7 \mathrm{~A} \mathrm{a}$ \\
\hline Glyphosate + Ureia & 1.440 & 5,0 & $99,0 \mathrm{~A} \mathrm{a}$ & $98,0 \mathrm{~A} \mathrm{a}$ & $98,0 \mathrm{~A} \mathrm{a}$ & $97,3 \mathrm{~A} \mathrm{a}$ \\
\hline Glufosinato de Amônio & 400 & -- & $45,0 \mathrm{~B} \mathrm{~b}$ & $83,3 \mathrm{C} \mathrm{a}$ & $41,7 \mathrm{~B} \mathrm{~b}$ & $90,3 \mathrm{~B} \mathrm{a}$ \\
\hline \multicolumn{3}{|c|}{$\mathrm{F}_{(\text {Trat } \mathrm{x} \text { Exp) }}$} & \multicolumn{2}{|c|}{$25,38 * *$} & \multicolumn{2}{|c|}{$99,85 * *$} \\
\hline \multicolumn{3}{|c|}{$\mathrm{DMS}_{\mathrm{Esp}}$} & \multicolumn{2}{|c|}{4,69} & \multicolumn{2}{|c|}{2,83} \\
\hline \multicolumn{3}{|c|}{ CV $(\%)$} & \multicolumn{2}{|c|}{3,32} & \multicolumn{2}{|c|}{1,98} \\
\hline
\end{tabular}

${ }^{1 /}$ Dose de glyphosate, em $\mathrm{g} \mathrm{ha}^{-1}$; ${ }^{2} / \mathrm{SA}$ - sulfato de amônio; * Médias seguidas por letras iguais, maiúsculas nas colunas e minúsculas nas linhas, não diferem entre si pelo teste de agrupamento de médias de Scott-Knott (coluna) ou teste 't' (linha), a 5\% de significância; ** Teste $\mathrm{F}$ significativo a $1 \%$. 
pela adição de sulfato de amônio se deve à maior absorção do herbicida pelas plantas (Costa \& Appleby, 1986; Satchivi et al., 2000). Isso resultaria em maior translocação do produto das folhas tratadas para toda a planta (Young et al., 2003). Segundo Nalewaja et al. (1992) e Thelen et al. (1995), o aumento da eficácia está relacionado com a formação de complexos de sais glyphosate-amônio, que são mais facilmente absorvidos pelas folhas, através da cutícula, que complexos glyphosatecálcio ou glyphosate-sódio.

O caráter ácido-fraco, hidrofilico e zwitteriônico do glyphosate sugere impermeabilidade da membrana celular ao produto para entrada nas células por simples difusão. Estudos propõem vias de penetração do glyphosate no simplasto por difusão passiva não-facilitada, por absorção ativa relacionada com o gradiente de $\mathrm{pH}$ através da membrana, ou mesmo por transportadores de fosfato. Young et al. (2003) comentam também que a acidificação da calda provocada pelo sulfato de amônio permitiria que mais moléculas permanecessem sob a forma não-dissociada, que atravessa a membrana plasmática com maior facilidade. Ruiter \& Meinen (1996) observaram que o sulfato de amônio elevou a absorção de glyphosate por protoplastos isolados de Elytrigia repens, com pequeno incremento no $\mathrm{pH}$ citoplasmático. No interior das células, em função do $\mathrm{pH}$ mais alcalino que o meio intercelular, as moléculas de glyphosate passariam novamente para a forma dissociada, atuando como herbicidas (Young et al., 2003).

Aos 28 DAA, não foram observados efeitos de diferenciação de dose ou de fonte nitrogenada, o que sugere que a adição de fontes nitrogenadas à calda de pulverização somente acelerou a morte das plantas, o que também ocorreu com a aplicação de glyphosate isolado a $720 \mathrm{~g} \mathrm{ha}^{-1}$ (Tabela 4). Em todas as avaliações, observou-se controle diferenciado do herbicida glufosinato de amônio sobre as duas espécies, tendo o apaga-fogo sido mais tolerante à dose de $400 \mathrm{~g} \mathrm{ha}^{-1}$ desse herbicida (Tabelas 3 e 4), o que está em coerência com a ausência de registro para $A$. tenella (Rodrigues \& Almeida, 2005).

Não foram observados incrementos de controle proporcionados pela adição das fontes nitrogenadas nas avaliações de massa fresca ou seca (Tabelas 5 e 6), porém a comparação das espécies manteve a caracterização do capim-massambará como menos sensível à

Tabela 5 - Massa fresca residual (\%) de apaga-fogo (ALRTE) e capim-massambará (SORHA), avaliada aos 28 dias após aplicação (DAA) dos tratamentos. Piracicaba, 2008

\begin{tabular}{|c|c|c|c|c|c|}
\hline \multirow{3}{*}{ Tratamento } & \multicolumn{2}{|c|}{ Dose } & \multirow{2}{*}{\multicolumn{3}{|c|}{ Massa fresca residual $\stackrel{2 /}{(\%)}$}} \\
\hline & \multirow{2}{*}{$\frac{\text { Herbicida }}{\left(\mathrm{g} \mathrm{ha}^{-1}\right)}$} & \multirow{2}{*}{$\frac{\mathrm{FN}}{\left(\mathrm{g} \mathrm{L}^{-1}\right)}$} & & & \\
\hline & & & ALRTE & SORHA & Média \\
\hline Testemunha sem aplicação & -- & -- & 100,0 & 100,0 & $100,0 \mathrm{C}$ \\
\hline Glyphosate $^{\underline{1 /}}$ & 720 & -- & 18,1 & 24,6 & $21,3 \mathrm{~A}$ \\
\hline Glyphosate + Sulfato de Amônio & 720 & 7,5 & 20,6 & 32,1 & $26,4 \mathrm{~A}$ \\
\hline Glyphosate + Sulfato de Amônio & 720 & 15,0 & 11,5 & 23,7 & $17,6 \mathrm{~A}$ \\
\hline Glyphosate + Ureia & 720 & 2,5 & 6,7 & 31,5 & $19,1 \mathrm{~A}$ \\
\hline Glyphosate + Ureia & 720 & 5,0 & 10,6 & 27,7 & $19,2 \mathrm{~A}$ \\
\hline Glyphosate & 1.440 & -- & 14,5 & 14,0 & $14,2 \mathrm{~A}$ \\
\hline Glyphosate + Sulfato de Amônio & 1.440 & 7,5 & 13,4 & 18,2 & $15,8 \mathrm{~A}$ \\
\hline Glyphosate + Sulfato de Amônio & 1.440 & 15,0 & 19,9 & 19,7 & $19,8 \mathrm{~A}$ \\
\hline Glyph osate + Ureia & 1.440 & 2,5 & 15,8 & 18,5 & $17,2 \mathrm{~A}$ \\
\hline Glyphosate + Ureia & 1.440 & 5,0 & 11,6 & 15,7 & $13,6 \mathrm{~A}$ \\
\hline Glufosinato de Amônio & 400 & -- & 83,4 & 57,2 & $70,3 \mathrm{~B}$ \\
\hline Média & \multicolumn{2}{|c|}{-} & $27,2 \mathrm{a}$ & $31,9 \mathrm{~b}$ & -- \\
\hline $\mathrm{F}_{(\text {Trat } x \text { Exp) }}=1,72^{\mathrm{NS}}$ & $\mathrm{CV}(\%)=21,20$ & \multicolumn{2}{|c|}{$\mathrm{F}_{\text {Trat }}=21,20 * *$} & \multicolumn{2}{|c|}{$\mathrm{F}_{\mathrm{Esp}}=7,23^{*}$} \\
\hline
\end{tabular}

1/ Dose de glyphosate, em $\mathrm{g} \mathrm{ha}^{-1}$; ${ }^{2 /}$ Dados previamente transformados por $\sqrt{x+0,5}$; Médias seguidas por letras iguais, maiúsculas nas colunas e minúsculas nas linhas, não diferem entre si pelo agrupamento de médias por teste de Scott-Knott (coluna) ou teste 't' (linha), a 5\% de significância; Ns Não-significativo; * Teste ' $\mathrm{F}$ ' significativo a $5 \%$; ** Teste $\mathrm{F}$ significativo a $1 \%$. 
Tabela 6 - Massa seca residual (\%) de apaga-fogo (ALRTE) e capim-massambará (SORHA), avaliada aos 28 dias após aplicação (DAA) dos tratamentos. Piracicaba, 2008

\begin{tabular}{|c|c|c|c|c|c|}
\hline \multirow{3}{*}{ Tratamento } & \multicolumn{2}{|c|}{ Dose } & \multirow{2}{*}{\multicolumn{3}{|c|}{ Massa seca residual * (\%) }} \\
\hline & \multirow{2}{*}{$\begin{array}{c}\text { Herbicida } \\
\left(\mathrm{g} \mathrm{ha}^{-1}\right)\end{array}$} & \multirow{2}{*}{$\begin{array}{c}\mathrm{FN} \\
\left(\mathrm{g} \mathrm{L}^{-1}\right)\end{array}$} & & & \\
\hline & & & ALRTE & SORHA & Média \\
\hline Testemunha sem aplicação & - & - & 100,0 & 100,0 & $100,0 \mathrm{~B}$ \\
\hline Glyphosate $^{\underline{1} /}$ & 720 & -- & 33,5 & 50,1 & $41,8 \mathrm{~A}$ \\
\hline Glyphosate + Sulfato de Amônio & 720 & 7,5 & 19,9 & 67,9 & $43,9 \mathrm{~A}$ \\
\hline Glyphosate + Sulfato de Amônio & 720 & 15,0 & 25,9 & 51,4 & $38,6 \mathrm{~A}$ \\
\hline Glyphosate + Ureia & 720 & 2,5 & 15,7 & 53,2 & $34,4 \mathrm{~A}$ \\
\hline Glyphosate + Ureia & 720 & 5,0 & 21,3 & 59,3 & $40,3 \mathrm{~A}$ \\
\hline Glyphosate & 1.440 & -- & 37,4 & 41,6 & $39,5 \mathrm{~A}$ \\
\hline Glyphosate + Sulfato de Amônio & 1.440 & 7,5 & 30,8 & 47,7 & $39,3 \mathrm{~A}$ \\
\hline Glyphosate + Sulfato de Amônio & 1.440 & 15,0 & 46,0 & 51,1 & $48,5 \mathrm{~A}$ \\
\hline Glyphosate + Ureia & 1.440 & 2,5 & 33,5 & 49,6 & $41,5 \mathrm{~A}$ \\
\hline Glyphosate + Ureia & 1.440 & 5,0 & 31,9 & 44,1 & $38,0 \mathrm{~A}$ \\
\hline Glufosinato de Amônio & 400 & -- & 60,7 & 71,2 & $65,9 \mathrm{~A}$ \\
\hline Média & & & $38,1 \mathrm{a}$ & $57,3 \mathrm{~b}$ & -- \\
\hline $\mathrm{F}_{(\text {Trat } x \text { Exp) }}=1,41^{\mathrm{NS}}$ & $=19,89$ & \multicolumn{2}{|c|}{$\mathrm{F}_{\text {Trat }}=5,00^{* *}$} & \multicolumn{2}{|c|}{$\mathrm{F}_{\operatorname{Exp}}=25,96^{* *}$} \\
\hline
\end{tabular}

${ }^{1 /}$ Dose de glyphosate, em $\mathrm{g} \mathrm{ha}^{-1}$; * Dados previamente transformados por $\sqrt{x+0,5}$; Médias seguidas por letras iguais, maiúsculas nas colunas e minúsculas nas linhas, não diferem entre si pelo agrupamento de médias pelo teste de Scott-Knott (coluna) ou teste ' $t$ ' (linha), a 5\% de significância; Ns Não-significativo; ** Teste ' $\mathrm{F}$ ' significativo a $1 \%$.

aplicação de herbicidas. Quanto à avaliação de massa fresca, o herbicida glufosinato de amônio apresentou controle diferenciado, com massa superior à do glyphosate e inferior à da testemunha sem aplicação (Tabela 5), o que está em concordância com os dados de controle.

Os mecanismos que promovem incrementos de controle relacionados com a adição de ureia ou sulfato de amônio à calda do glyphosate ainda não estão completamente esclarecidos. Todavia, observou-se que a adição de fontes nitrogenadas acelerou a morte da espécie mais tolerante, com controle diferenciado em relação à aplicação de somente glyphosate na dose de $720 \mathrm{~g} \mathrm{ha}^{-1}$. A aplicação de glyphosate com sulfato de amônio e ureia mostrou potencial de utilização em áreas agrícolas brasileiras, principalmente na ocorrência de água dura, espécies de maior tolerância a herbicidas ou possibilidade de chuvas em curto intervalo de tempo. No entanto, mais estudos devem ser conduzidos visando a diferenciação de resposta das espécies às combinações de herbicida-fonte nitrogenada, bem como à dose do fertilizante a ser adicionada.

\section{AGRADECIMENTOS}

Os autores agradecem ao Conselho Nacional de Desenvolvimento Científico e Tecnológico (CNPq), pela bolsa de doutorado concedida ao primeiro, segundo e quinto autores.

\section{LITERATURA CITADA}

CHRISTOFFOLETI, P. J. et al. Glyphosate sustainability in South American cropping systems. Pest Manag. Sci., v. 64, n. 4, p. 422-427, 2008.

COSTA, J.; APPLEBY, A. P. Effects of ammonium sulphate on leaf growth inhibition by glyphosate in Cyperus esculentus L. Crop Protec., v. 5, n. 5, p. 314-318, 1986.

DURIGAN, J. C. Efeito de adjuvantes na calda e no estádio de desenvolvimento das plantas daninhas no controle do capim-colonião (Panicum maximum) com glyphosate. Planta Daninha, v. 10, n. 1/2, p. 39-44, 1992

FRANSON, M. A. H. Standard methods for the examination of water and wastewater. 19.ed. Washington: American Public Heath Association, 1995 $1074 \mathrm{p}$ 
GAUVRIT, C. Glyphosate response to calcium, ethoxylated amine surfactant, and ammonium sulfate. Weed Technol., v. 17, n. 4 , p. $799-804,2003$

HALL, G. J.; HART, C. A.; JONES, C. A. Plants as sources of cations antagonistic to glyphosate activity. Pest Manag. Sci., v. 56, n. 4, p. 351-358, 2000.

JAKELAITIS, A. et al. Controle de Digitaria horizontalis pelos herbicidas glyphosate, sulfosate e glyphosate potássico submetidos a diferentes intervalos de chuva após a aplicação. Planta Daninha, v. 19, n. 2, p. 279-285, 2001.

MaciSAAC, S. A.; PAUL, R. N.; DEVINE, M. D. A scanning electron microscope study of glyphosate deposits in relation to foliar uptake. Pestic. Sci., v. 31, n. 1, p. 53-64, 1991.

MONQUERO, P. A. et al. Absorção, translocação e metabolismo do glyphosate por plantas tolerantes e suscetíveis a este herbicida. Planta Daninha, v. 22, n. 3, p. 445-451, 2004.

MOREIRA, M. S.; CHRISTOFFOLETI, P. J. Resistência de plantas daninhas aos herbicidas inibidores da EPSPs (Grupo G). In: CHRISTOFFOLETI, P. J. (Coord.). Aspectos de resistência de plantas daninhas a herbicidas. 3.ed. Piracicaba: HRAC-BR, 2008. p. 78-96.

MUELLER, T. C. et al. Comparison of glyphosate salts (isopropylamine, diammonium, and potassium) and calcium and magnesium concentrations on the control of various weeds. Weed Technol., v. 20, n. 1, p. 164-171, 2006

NALEWAJA, J. D.; MATYSIAK, R. Salt antagonism of glyphosate. Weed Sci., v. 39, n. 4, p. 622-628, 1991.

NALEWAJA, J. D.; MATYSIAK, R.; FREEMAN, T. P. Spray droplet residual of glyphosate in various carriers. Weed Sci., v. 40, n. 4, p. 576-589, 1992

NALEWAJA, J. D.; MATYSIAK, R. Optimizing adjuvants to overcome glyphosate antagonistic salts. Weed Technol., v. 7, n. 2, p. 337-342, 1993.

NORSWORTHY, J. K.; BURGOS, N. R.; OLIVER, L. R. Differences in weed tolerance to glyphosate involve different mechanisms. Weed Technol., v. 15, n. 4, p. 725-731, 2001.

NURSE, R. E. et al. Annual weed control may be improved when AMS is added to below-label glyphosate doses in glyphosate-tolerant maize (Zea mays L.). Crop Protect., v. 27, n. 3 , p. $452-458,2008$.
PEDRINHO JÚNIOR, A. F. F. et al. Momento da chuva após a aplicação e a eficácia dos herbicidas sulfosate e glyphosate aplicados em diferentes formulações. Planta Daninha, v. 20, n. 1, p. 115-123, 2002.

RAMSDALE, B. K.; MESSERSMITH, C. G.; NALEWAJA, J. D. Spray volume, formulation, ammonium sulfate, and nozzle effects on glyphosate efficacy. Weed Technol., v. 17, n. 3, p. 589-598, 2003.

RODRIGUES, B. N.; ALMEIDA, F. S. Guia de herbicidas. 5.ed. Londrina: 2005. 592 p.

RUITER, H. D.; MEINEN, E. Adjuvant-increased glyphosate uptake by protoplasts isolated from quackgrass Elytrigia repens (L.) Nevski. Weed Sci., v. 44, n. 1, p. 38-45, 1996.

SALISBURY, C. D.; CHANDLER, J. M.; MERKLE, M. G. Ammonium sulfate enhancement of glyphosate and SC-0224 control of johnsongrass (Sorghum halepense). Weed

Technol., v. 5, n. 1, p. 18-21, 1991

SATCHIVI, N. M. et al. Absorption and translocation of glyphosate isopropylamine and trimethysulfonium salts in Abutilon theophrasti and Setaria faberi. Weed Sci., v. 48, n. 6 , p. $675-679,2000$.

SCOTT, A. J.; KNOTT, M. A. Cluster analysis method for grouping means in the analysis of variance. Biometrics, v. 30, n. 3, p. 507-512, 1974.

SHEA, P. J.; TUPY, D. R. Reversal of cation-induced reduction in glyphosate activity with EDTA. Weed Sci., v. 16, n. 1, p. 1-6, 1984.

SUWUNNAMEK, U.; PARKER, C. Control of Cyperus rotundus with glyphosate: the influence of ammonium sulfate and other additives. Weed Res., v. 15, n. 1, p. 13-20, 1975.

THELEN, K. D.; JACKSON, E. P.; PENNER, D. The basis for the hard-water antagonism of glyphosate activity. Weed Sci., v. 43, n. 4, p. 541-548, 1995.

WILLS, G. D.; McWHORTER, C. G. Effect of inorganic salts on the toxicity and translocation of glyphosate and MSMA in purple nutsedge (Cyperus rotundus). Weed Sci., v. 33, n. 6, p. $755-761,1985$.

YOUNG, B. G. et al. Glyphosate translocation in common lambsquarters (Chenopodium album) and velvetleaf (Abutilon theophrasti) in response to ammonium sulfate. Weed Sci., v. 51, n. 2 , p. $151-156,2003$. 